\title{
Scale Invariant Fractional Shape matching for computerized images
}

\author{
Rashmi Ghongade $^{1}$, Dr.U.M.Gokhale ${ }^{2}$, Prof.N.H.Pandey ${ }^{3}$ \\ Department of Electronics Engineering G. H. Raisoni Institute of Engineering and Technology for Women \\ Nagpur, India \\ Department of Electronics Engineering G. H. Raisoni Institute of Engineering and Technology for Women \\ Nagpur, India \\ Department of Electronics Engineering G. H. Raisoni Institute of Engineering and Technology for Women \\ Nagpur, India
}

\begin{abstract}
Shape Matching is an important aspect in image processing . It is an important step whenever there arouses a need to match a certain image with another. There are a lot of applications wherein the image matching is the key step towards the fulfillment of the application. So in such cases Partial image matcing usually becomes a crucial part of the whole process. Hence here we present a novel approach of partial shape matching for the scale invariant images. The problem of establishing a match between an open contour and a closed contour is tried to resolve in this paper. The computation of the shape descriptors is alo done along open or closed contours. The net ordered collections of shape descriptors constitute the global shape representation. Here we propose an alternate technique to an existing Dynamic time warping matching technique for the matching of the shape representation. Use of the properties of the employed shape descriptor, sampling scheme and matching procedure, the proposed approach performs partial shape matching that is invariant to Euclidean transformations. Also the problem of matching the closed to closed contours is automatically treated as a special case.

The proposed method gives a better vision and a better result as compared to that of existing system because of the extensive dataset used in the proposed application. And thus the proposed system is a better solution of partial shape matching and the problems related to partial shape matching
\end{abstract}

Keywords: - Partial shape matching, shape descripto, DTW(dynamic time warping technique)r.

\section{INTRODUCTION}

The most common problem faced in pattern recognition is shape matching. It is nothing but the problem of describing a shape and calculating its similarity to others. In general, when too much noise exists in the shape, the performance of existing shape matching techniques tends to drop significantly. Because of occlusion or segmentation errors, it may be the case that only parts of objects in a given image have correct contours. Therefore, a shape similarity measure based on parts of objects is needed.The identification of shapes given their parts is still an unsolved problem in shape similarity. Shape matching is a well investigated problem in computer vision and has versatile applications as e.g. in object detection or image retrieval. The choice of the shape representation has a significant effect on the matching step.It is the most impoetant step. Basically, Shape matching deals with transforming a shape, and measuring the resemblance with another one,using some similarity measure. So, shape similarity measures are an essential ingredient in shape matching.

Shape matching is of central importance in a number of computer vision problems such as shape classification, retrieval, recognition, and simplification. This system deals with the partial shape matching of binary images only. [5] In most of the cases, arbitrary differences in scale and Orientation should not affect the matching process. Due to viewpoint dependencies and shape articulations and deformations, different 2D image projections of the shape of the same 3D object may differ considerably. Further complications are caused by occlusions which force shape matching to be based on partial evidence.

In some case, the best matching of an open contour with part of a closed contour needs to be established [4] but all of the above complicating factors contribute collectively to increasing the complexity of the matching problem. Shape matching including wide range of applications such as, object detection and recognition, content based retrieval of images and image registration.To perform shape matching, most of the existing methods [5] define shape representations and descriptors which are then compared through appropriately selected methods and metrics. It may arise some serious problems as, length problem, scale problem, and distortion problem.None of current shape matching techniques provides solutions to all problems listed above. Even feature-based approaches, although potentially being based on local features, require the presence of most of the object to compute the statistics of the features.The existing partial shape similarity 
measures require that the query part is nearly identical to the corresponding part of the target contour, which is clearly an unrealistic assumption due to noise distortions and due to (even small) perspective projection changes. Veltkamp and Tanase [7] proposed to use an extended dynamic programming approach directly on the turn angle function representation of object contours. Since their matching of a single part is not elastic, their approach is not scale invariant.

\section{RELATED WORK}

For carrying out the study of the desired topic, the detailed study of the related work done by other authors needed to be studied. We briefly take an overview of the existing approaches on the partial shape matching. Basically shape matching is such a problem which has been discussed in many research.Torres and falco [6] have computed the image skeleton at different scales and further used them to detect the minute details of the contour of the image. There are many researches based on the image skeletonization.In [14] Sebastian has presented a technique based on the notion of shok graphs.It says that the shapes that possess same shok graphs are equivalent in nature. Some methods instead of relying on the skeleton technique have chosed to method based on representation and properties of all points of any shape. Gorelick[15] propose the representation of the interior points by the distance that a person will travel before reaching it,provided that starting point of his journey is located on shape's silhouette. Ebrahim [12] made a research based on Hilbert curve. He presented a method that transforms the rastor of each shape into a one dimensional signal according to the occurance of the shape points on the Hilbert curve. In some case, the best matching of an open contour with part of a closed contour needs to be established [2] but all of the above complicating factors contribute collectively to increasing the complexity of the matching problem. Shape matching including wide range of applications such as, object detection and recognition, content based retrieval of images and image registration.To perform shape matching, most of the existing methods [1] define shape representations and descriptors which are the compared through appropriately selected methods and metrics. The existing partial shape similarity measures [2] require that the query part is nearly identical to the corresponding part of the target contour, which is clearly an unrealistic assumption due to noise distortions and due to (even small) perspective projection changes. In the context of this work, we are interested in addressing the $2 \mathrm{D}$ shape matching problem by simultaneously considering all the above complicating factors. Shapes are represented as binary images depicting foreground objects over their background. Based matching technique [10] that accomplishes global 2D shape matching based on the computed shape descriptors. The key novelty in this variant is its ability to handle partial matching. Although primarily developed for matching open to closed contours, the proposed scheme treats the Matching of two closed contours as a special case. This can be effectively achieved by treating one of the two closed contours as an open one that starts and ends at the same point. Latecki et al. in [10] propose a method for shape matching based on dynamic programming. A particularly interesting aspect of this method is that it addresses the partial shape matching problem. More specifically, the method is able to establish the best match between an open silhouette and a part of a closed silhouette. Purpose of shape description. Like in the case of DTW, MVM reduces the problem of optimal alignment between two sequences to a shortest path problem in a Directed Acyclic Graph (DAG), which can be efficiently solved using Dynamic Programming. The key difference between MVM and DTW is the number of connections allowed in each Node of the DAG, corresponding to different matching types in the original problem.

\section{PROPOSED APPROACH}

Some standard steps are followed and implemented thereby trying to get the desired results. Here in this paper the boundary points are the main aspect on which shape matching is done.Basically a similarity between the shapes and the shape ailments are computed.

FLONACHART

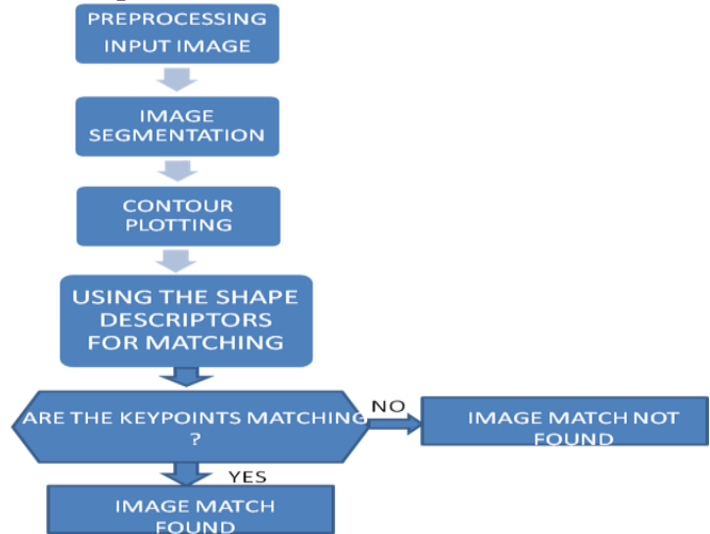

Fig3.1. Flowchart of the proposed system 


\section{IMAGE ANALYSIS}

Automatic recognition of image cells usually consists of four major steps, including: Preprocessing ,Image segmentation,Contour plotting,Feature extraction and Classification. The detail study of the process is done by considering the points below

3.1 Preprocessing: The process of Preprocessing is basically done to make the image ready for any further process on it.In the preprocessing the unwanted noise or the blurring of image is removed so that the image wchich is considered for the computational operation is noise free. In this module first we will load the binary images,then Gaussian filter will be applied for input images to remove any unwanted noise in the image.

\subsection{Shape Representation:}

At a first step, a given silhouette is uniformly sampled and one descriptor is computed on each point sample. The descriptor consists of the distances of the particular point from the closest silhouette points, along equiangular directions defined in the inner part of the shape. The median value of these distances provides an estimate of local scale.

\subsection{Contour Extraction}

The input to the proposed method is a binary image containing a foreground object. Shape description and matching require consistency with respect to this order. Therefore, lists of contour points are reversed, depending on the sign of the area covered by a silhouette and the convention that this must be positive. This convention ensures that the points that points of all the compared shapesare ordered in counter clockwise direction.

We proceed by performing a fixed sub sampling of the silhouette by retaining one out of pixels. In order to eliminate small amounts of quantization noise, prior to sub sampling, Gaussian smoothing of the silhouette is performed with a Gaussian kernel of variance.

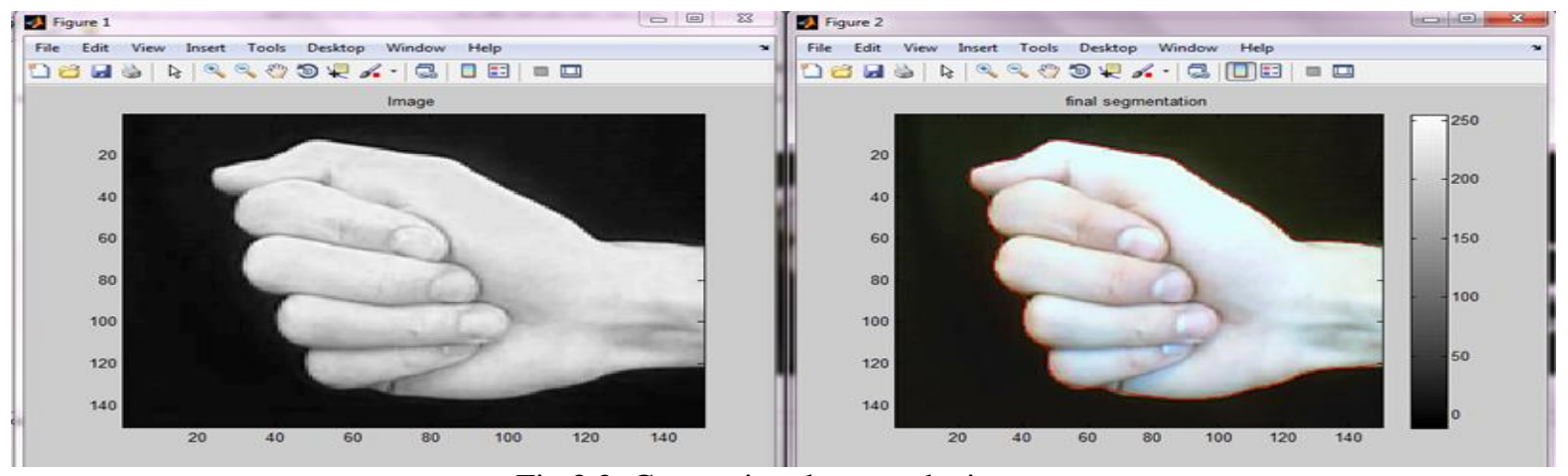

Fig.3.2. Contouring done on the image

\subsection{Local Shape Descriptors}

The idea behind the proposed descriptor lies on measuring the distance of a certain silhouette point from the closest points of the same silhouette, along properly defined directions be a point on a silhouettes for which a local shape descriptor should be computed.

\subsection{Local scale estimation}

An estimate of the local scale $\mathrm{S}\left(\mathrm{s}_{\mathrm{i}}\right)$ of a silhouette point $\mathrm{s}_{\mathrm{i}}$ can be computed as the mean of the finite distances of a descriptor:

$$
S\left(s_{i}\right)=\frac{1}{\left|F_{i}\right|} \sum_{j \in F_{i}} l_{i j},
$$

where $\mathrm{Fi}=\{\mathrm{lij} \in \mathrm{d}(\mathrm{si}): \mathrm{lij} \neq 1 \infty \wedge \wedge \mathrm{lij} \neq 1 \mathrm{U}\}$ and $|$.$| denotes set cardinality. The intuition behind the particular$ representation of local scale is that $\mathrm{S}(\mathrm{si})$ is indeed proportional to the level of detail of local shape.thedescriptors and scale estimatescan be computed for all points in s. From The various experiments it can be shown that increased accuracy wchich is obtained is not woth of the extra computayion that has to be carried out.

Scale adjusted sampling:

Given $\mathrm{S}\left(\mathrm{S}_{\mathrm{i}}\right)$, local scale estimates for each point $\mathrm{S}_{\mathrm{i}}$ in $\mathrm{s}$, we can sample $\mathrm{s}$ in local, scale-dependent way. Points of $s$ are selected consecutively based on the estimated local scale. For a given sampled point, the points of $\mathrm{s}$ to be discarded immediately after it, are proportional in number to the local scale of the point, and the point 
following them comprises the next position to sample. More specifically, a local sampling offset o is chosen proportional to the local scale, or equivalently, inversely proportional to local detail.
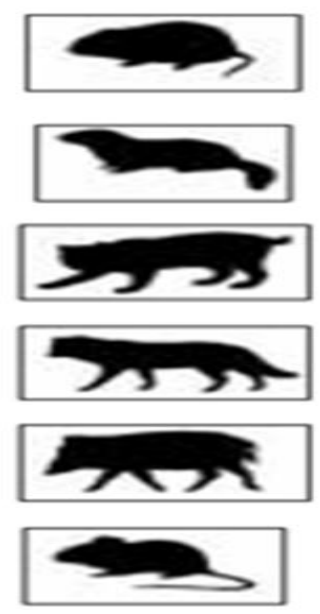

Fig 3.3 Mammal silhouette: column1: query image.
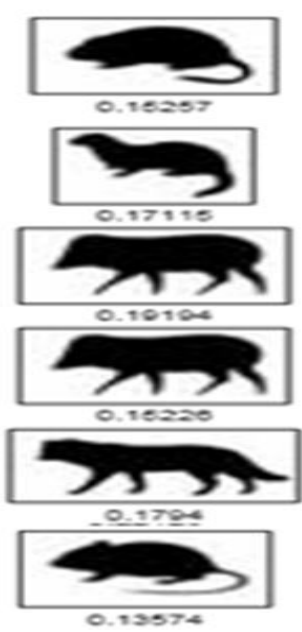

Column2: matching images, sorted by similarity

\subsection{Edge detection:}

As we uses the boundary basedrepresentation, the outer boundary of the shape only is considered. This is done by describing the considered region using external characteristics i.e. edges of the image. For the extraction of the shape of the shape of the image, first we identify the object of the shape, second detecting edges and extracting the feature of the object, later applying the sampling.

Figure 3.4: Extracting feature the shape An example of extracting feature of shape is shown in above figure, passing binary image is shown in (a) then detecting object and extracting the edges is shown in (b), (c) and after applying down sapling is shown in (d).

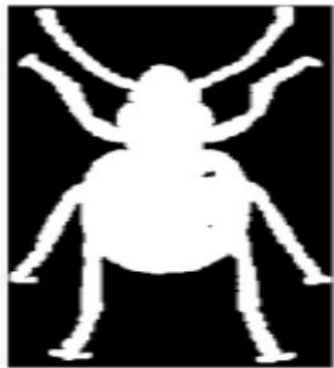

$\mathrm{b}$

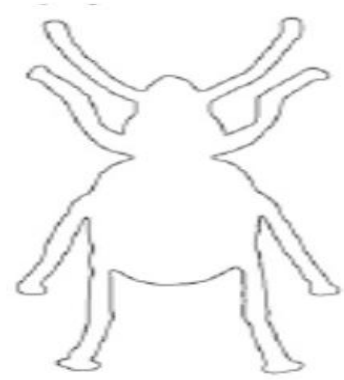

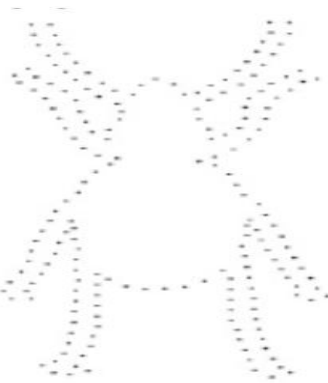

$\mathrm{c}$

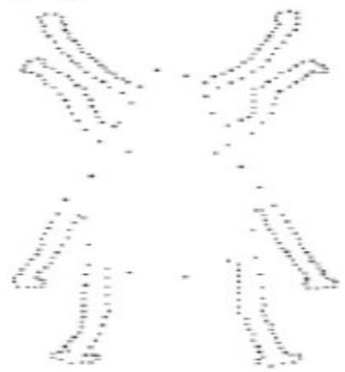

d

\subsection{Matching technique:}

In the shape matching we will first compare the shape descriptors to the given input image data to see if the parameters of the two images match and if they match, then to what extend.

Let $\mathrm{d}(\mathrm{sx})=\{1 \mathrm{x} 1, \mathrm{~lx} 2, \ldots, \mathrm{lxk}\}, \mathrm{d}(\mathrm{ty})=\{\mathrm{ly} 1, \mathrm{ly} 2, \ldots, \mathrm{lyk}\}$ be two shape descriptors at points sx and ty, respectively. The goal is to establish a distance measure $\mathrm{D}(\mathrm{sx}, \mathrm{ty})$ between the descriptors $\mathrm{d}(\mathrm{sx})$ and $\mathrm{d}(\mathrm{ty})$. $\mathrm{D}(\mathrm{sx}, \mathrm{ty})$ is defined based on the pairwise comparison of the descriptors' coordinates, according to:

$$
\mathrm{P}\left(s_{x}, t_{y}\right)=\frac{1}{k} \sum_{i=1}^{k} \Delta\left(l_{x i}, l_{y i}\right)
$$

where $\Delta(. .$,$) is a function that compares its arguments and returns a value in the range [0..1]. As The$ Dynamic Time Warping (DTW) is a wellknown technique in many areas. It is used for measuring the similarity between given shapes. It is a technique for measuring similarity between two sequences which may vary in time or speed. DTW allows a computer to find an optimal match between two given sequences (e.g. time series) with certain restrictions. DTW can compare characters in a way that is similar to the way humans compare characters, or at least generates the same results. 


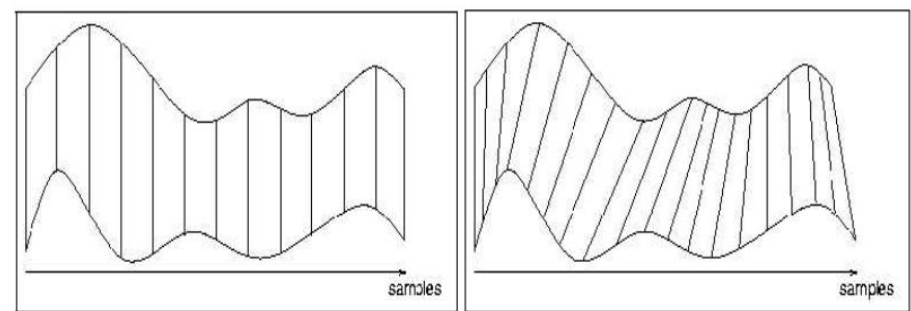

(a) one to one comparison (b) DTW comparison

Figure 3.5: Comparison of two curves using one to one comparison and Dynamic Time Warping

\subsection{Shape matching:}

In shape matching the shape of the given input image is compared with the shapes of the answere images using the dynamic time warping technique. Matching open contours against parts of closed contours i.e., the extracted edge of given input image is then matched with that of the answer images. And the shape matching is carried out by applying DTW technique. Matching must be done in the presence of noise i.e. geometrical distortion. The goal of the matching step is to estimate the similarity of two given contours based on the descriptors already computed on them. This is achieved by establishing correspondences between contour points. The matching of open against that of the closed contours is similarly treated. For instance, Assume an open contour s that needs to be matched with part of a closed contour t. We capitalize on the observation that this can be computed as the best match between $\mathrm{s}$ and any substring of length less than $\mathrm{Nt}$ from $\mathrm{t} \bigoplus \mathrm{t}$, i.e. the concatenation of $t$ with itself. The duplication of the target string ensures that the source string $s$ can be matched with the target string without having to wrap around at string ends. Then, the problem is transformed into one of searching for minimum cost paths in a directed acyclic graph and employ dynamic programming techniques that prune the search space by exploiting previously computed paths. Care is taken so as to enforce the constraint that the source, open string $\mathrm{s}$ cannot match a substring of $\mathrm{t} \oplus \mathrm{t}$ that has length greater than Nt; This would mean that $\mathrm{s}$ wraps around the cyclic string $\mathrm{t}$.

\section{CONCLUSION}

An Automated tool has been developed for the partial shape matching of any image, which will compare the input image to that of data set and show the results accordingly. The proposed automation tool thereby has many advantages and applications which can be useful for any computer aided tool.

The proposed system has many applications like

- Industrial inspection

- Fingerprint matching

- Object detection and recognition

- Stereo matching and content based image retrieval.

The proposed tool has been made on the Matlab 10 software on the Graphical user interface.So the results can be seen on the GUI frontpage of the matlab software. The snapshots of the frontpage of the GUI results are given below.

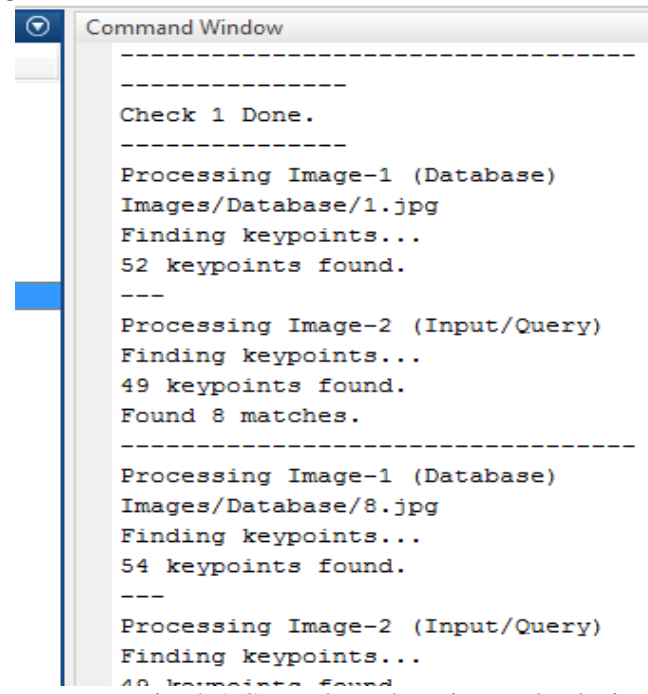

Fig 4.1 Snapshot showing calculation of the matched keypoints of the image 


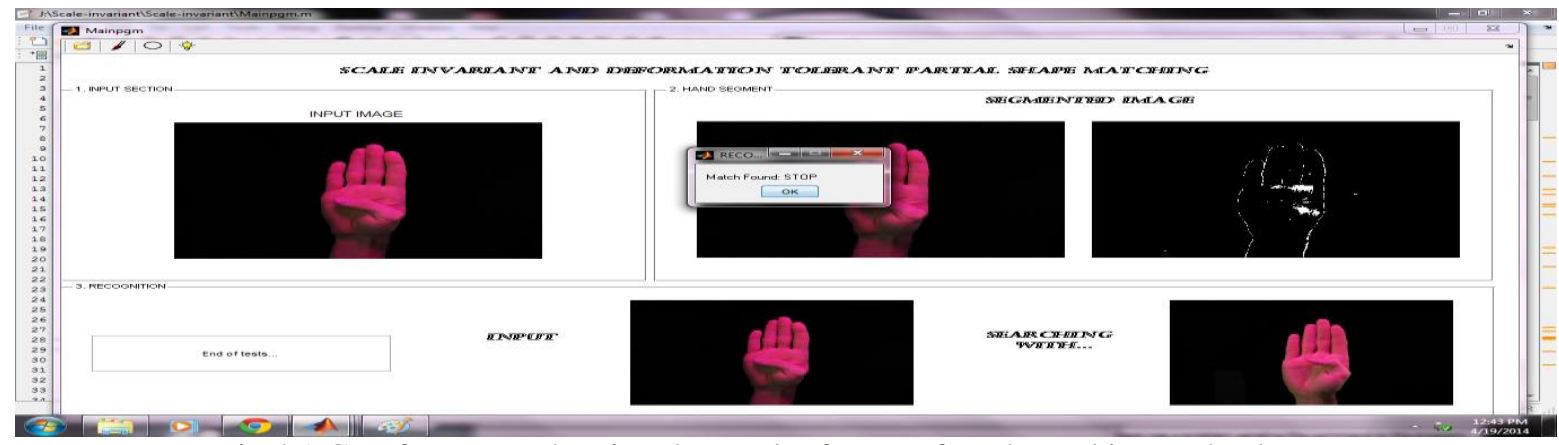

Fig 4.1 GUI frontpage showing the result of Image found matching to the dataset.

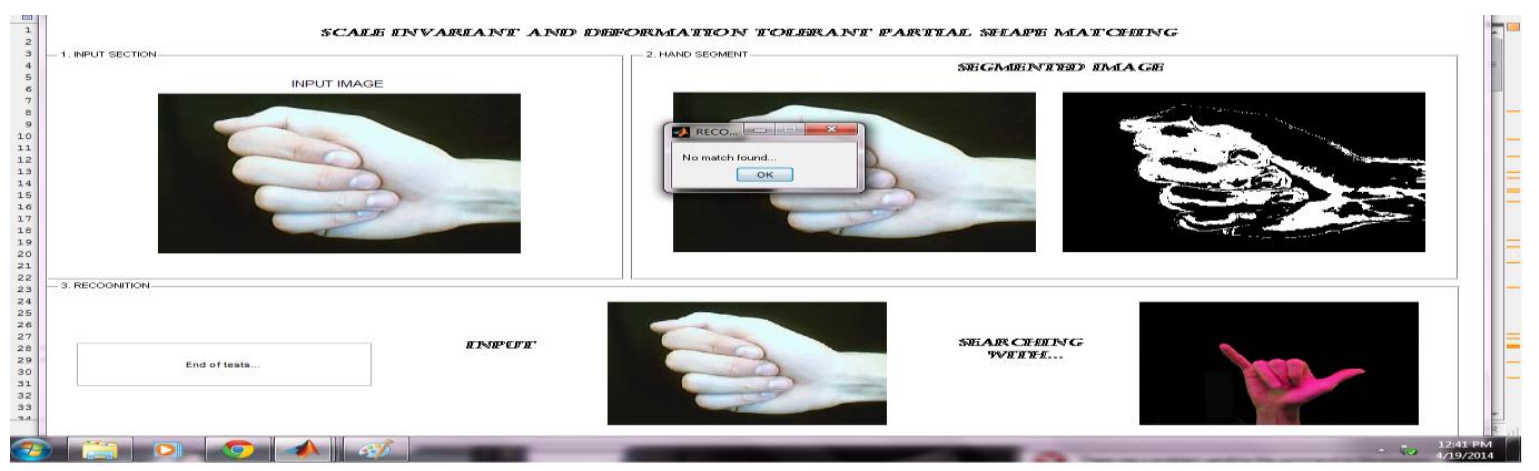

Fig 4.2 GUI frontpage showing the result of Image not found matching to the dataset.

\section{Journal Papers:}

\section{REFERENCES}

[1] A.R. Backes, D. Casanova, O.M. Bruno, A complex network-based approach for boundary shape analysis, Pattern Recognition 42 (1) (2009) 54-67

[2] M. Cui, J. Femiani, J. Hu, P. Wonka, A. Razdan, Curve matching for open 2d curves, Pattern Recognition Letters 30 (1) (2009) 1-10.

[3] T. Adamek, N.E. O'Connor, A multiscale representation method for nonrigid shapes with a single closed contour, IEEE Transactions on Circuits and Systems or Video Technology 14 (5) (May 2004) 742-753.

[4] R. da S. Torres, A.X. Falcão, Contour salience descriptors for effective image retrieval and analysis, Image and Vision Computing Journal 25 (1) (2007) 3-13.

[5] T.B. Sebastian, P.N. Klein, B.B. Kimia, Recognition of shapes by editing shock graphs, IEEE International Conference on Computer Vision, 2001, pp. 755-762.

[6] L. Gorelick, M. Galun, E. Sharon, R. Basri, A. Brandt, Shape representation and classification using the poisson equation, IEEE Transactions on Pattern Analysis and Machine Intelligence 28 (12) (2006) 1991-2005.

[7] Y. Ebrahim, M. Ahmed, W. Abdelsalam, S.C. Chau, Shape representation and description using the Hilbert curve, Pattern Recognition Letters 30 (4) (2009) 348-358.

[8] R. Basri, L. Costa, D. Geiger, D. Jacobs, Determining the similarity of deformable shapes, Vision Research 38 (1998) 135-143..

[9] N. Arica, F.T.Y. Vural, A perceptual shape descriptor, Proceedings of the $16^{\text {th }}$ International Conference on Pattern Recognition (ICPR'02), vol. 2, IEEE Computer Society, 2002, pp. 375-378.

[10] H. Ling, W.W. Jacobs, Shape classification using the inner-distance, IEEE Transactions on Pattern Analysis and Machine Intelligence 29 (2) (Feb. 2007) 286-299

[11] H. Sakoe, S. Chiba, A dynamic programming approach to continuous speech recognition, Proceedings of the 7th Int'1 Congress on Acoustics, Budapest, 1971.

[12] M. Vlachos, M. Hadjieleftheriou, D. Gunopulos, E. Keogh, Indexing multidimensional time-series with support for multiple distance measures, Proceedings of the 9th ACM SIGKDD Int'l Conference on Knowledge Discovery and Data Mining, ACM, New York, NY, USA, 2003, pp. 216-225.

[13] F.R. Schmidt, D. Farin, D. Cremers, Fast matching of planar shapes in sub-cubic runtime, Proceedings of the IEEE International Conference on Computer Vision, IEEE, 2007, pp. 1-6.

[14] P.F. Felzenszwalb, J.D. Schwartz, Hierarchical matching of deformable shapes, IEEE Conference on Computer Vision and Pattern Recognition, June 2007, pp. 1-8.

Books:

[15] Digital Image Processing Using MATLAB by Gonzalez 2nd edition. Published by Pearson Education 